Chapitre 1

\title{
Le «baston de berger» et la colonne triomphale
}

La thérapie est représentée dans La Bergerie de 1565 non par le baume qu'on trouve dans le Dictamen metrificum, mais par quelque chose qui est très concret et en même temps liminal: un bâton, un prétendu «baston de berger» qui sert non pas au combat mais au rétablissement de la santé mentale de ceux qui en usent. Il possède des pouvoirs quasi miraculeux, mais en même temps une fonction pratique. Il remplace les flèches d'Éros et celles des hommes de guerre, puisqu'il a une mission apaisante et pacifique, et il rappelle par sa forme d'autres bâtons légendaires, tels que celui d'Esculape qui symbolisait le pouvoir de la médecine, le caducée de Mercure - devenu de nos jours emblème des médecins et des pharmaciens -, le thyrse, qui fascinera Charles Baudelaire ${ }^{39}$, le trident de Neptune, le bâton d'Aaron, le bâton de Joseph qui fit venir les Hébreux en Égypte (Ézéchiel 37, v. 16-20), la canne de Joseph dans le livre protoévangélique des Actes de Jacques - la seule canne parmi celles des veufs palestiniens à se couvrir soudain de fleurs, pour indiquer qu'il était l'élu de Dieu, destiné à épouser la Vierge Marie -, le bâton tutélaire, la houlette du Psaume 23. Dans des ouvrages plus récents on trouve par exemple la lance magique de Bradamante, ou le bâton de la croix de Frère Jan dans Gargantua (exemples bien moins pacifiques que ce que décrit Belleau). Le lecteur est par conséquent prêt à attribuer des propriétés merveilleuses à la baguette qu'il trouve dans $L a$ Bergerie.

L'apparence à première vue utilitaire de l'instrument de Belleau, instrument fréquemment associé à l'autorité, au commandement, convient bien à un texte qui dépeint un petit monde très discipliné dirigé par Antoinette de Bourbon. Belleau y trouve place lorsque l'éducation de Charles d'Elbeuf lui est confiée ${ }^{40}$. Les membres de cette communauté de Joinville, qui cultivent les arts et contemplent la beauté de la nature, se sentent soutenus en temps de guerre. La famille des Guises est un de leurs appuis; son importance, ainsi que la continuité qu'elle représente, sont mises en valeur par l'évocation méticuleuse de tombeaux, la récitation d'épitaphes, et la description de festivités diverses.

39 «Le Thyrse», Le Spleen de Paris, repris en 1864 sous le titre Petits Poèmes en prose. Le morceau est dédié à Franz Liszt.

40 Voir Alexandre Eckhardt, Remy Belleau: sa vie - sa «Bergerie», p.76-89. 
Le bâton s'insère dans le cadre d'une représentation d'œuvres d'art et de baisers qui montre la communauté vaquant à des occupations paisibles. Il a comme parallèle une colonne commémorative et est intimement lié aux réflexions de Belleau sur la mémoire.

Une description détaillée est consacrée au bâton (II, p. 109-11 et 118-19)41. Elle suit l'ekphrasis d'une coupe de bois et fait penser, dans sa précision, à des ouvrages illustrés tels que Le Cosmolabe, ou Instrument Universel, concernant Toutes Observations qui se peuvent faire Par les Sciences Mathematiques, Tant au Ciel, en la Terre, comme en la Mer, par Jacques Besson (Paris: Ph. G. de Roville, 1567). Cet ouvrage minutieux et instructif nous rappelle que Belleau écrit à un moment où les instruments sont en train de prendre de l'importance dans les sciences. Le bâton de Belleau rappelle aussi le médecin Jean Fernel (très sensible à la mesure) et la manière dont il emploie des notions géographiques pour décrire le corps humain ${ }^{42}$.

Tout en étant un étui pour peintre, musicien ou architecte, le bâton de Belleau indique - entre autres choses - les heures (permettant sans doute d'organiser le temps aussi rigoureusement que le fait Antoinette de Bourbon dans La Bergerie), les signes du zodiaque, et les points cardinaux, et il peut se transformer en quatre flûtes qu'il fournit à un orchestre improvisé, créant ainsi l'harmonie musicale considérée depuis la plus haute antiquité comme fort thérapeutique. Le bâton peut contenir diverses choses, que le narrateur énumère, et servir à mesurer, ainsi que l'indique le «gentil artizan venu de la rive $d^{\prime} \vee$ vigne»:

Il me montra aussi comme on trouvoit aisement la demie toise sur le dos de ce bâton, qui contient trois pieds, chacun pié douze pouces, chacun pouce douze onces ou lignes, les marques en sont d'Ivoire sur le bois d'Ebene, de ces trois pieds on en fait la toise qui est de six, on en fait la coudee qui est d'un pié et demy, la perche doublant la demie toise huit fois, de l'autre costé on y trouve l'aune comme de Paris, de Lyon, de Provins, la canne et la brasse. (p. 119, l. 7-17)

41 Voir la postface de Marie Madeleine Fontaine, CEuvres poétiques, II, p. 261-64. Elle croit que le bâton était peut-être utilisé par Belleau «dans les différentes missions de signatures d'actes notariés que lui confia le marquis d'Elbeuf» (p. 263).

42 On consultera l'article intéressant de Bruno Lavillatte, «Lire le corps comme on lit une carte: l'anatomie 'géographique' dans la Physiologie de Jean Fernel», Revue des Amis de Ronsard, 24 (2011), p. 43-57. Voir aussi Théâtre de l'anatomie et corps en spectacle: Fondements d'une science de la Renaissance, actes du colloque Les Théâtres de l'anatomie Haïfa, 17-19 décembre 2002, éd. Ilana Zinguer et Isabelle Martin (Berne: Peter Lang, 2006). 
Avec sa série vertigineuse de chiffres, cette phrase donne l'impression que tout peut être mesuré, même si la mesure varie d'un endroit à l'autre. Le passage entier consacré à ce bâton témoigne du désir de se situer exactement dans le temps et dans l'espace, ainsi que de l'équipement compliqué mais compact nécessaire pour ce faire. Il faut des marques gravées qui préservent la mémoire des générations précédentes. En temps de guerre, de tels points de repère tendent à disparaître et les croyances à être ébranlées; mais l'évocation du bâton avec sa précision mathématique nous encourage à nous rappeler qui nous sommes, et quels sont les aspects essentiels de notre vie. Après tout, le bâton polyvalent peut servir de canne, «peut servir pour aller par païs, et pour s'apuyer étant bien ferré par le bout d'embas, et bien encorné d'une belle corne de Dain» (p. 119, l. 17-20). Il représente un ferme appui, une certitude non seulement mathématique mais philosophique, et la possibilité de marcher sain et sauf.

\subsection{Un moyen mnémotechnique}

Qui plus est, l'épisode du bâton merveilleux rend concrets quelques aspects de l'activité de l'écrivain et de celle de la mémoire, qui emmagasine des faits susceptibles d'être remémorés pour retrouver la gaieté. Le lecteur apprend que le bâton peut contenir des outils que le narrateur énumère et qui servent à dessiner avec précision:

Le creux de la pommelle peut servir à mettre creons $\&$ peintures liquides, \& celuy des fleutes à mettre plumes pinçeaux [sic], compas, équierre, papier, pour designer paisages, villes, chasteaux, \& bastimens rustiques. (p. 118, l. 7- p.119, l. 2)

Le bâton aide à noter exactement ce qui entoure son utilisateur, à en conserver la trace. De façon analogue, une des activités du poète consiste à s'efforcer de représenter avec sa plume quelques-uns des aspects de son expérience.

L'extrait concernant le bâton attire notre attention sur l'importance des bâtiments, étroitement liés aux procédés mnémotechniques employés à l'époque, puisque l'image d'un édifice complexe était utilisée dans les arts de la mémoire comme moyen de mémoriser des faits ou des personnes. L'on sait que d'après la Rhetorica ad Herennium, par exemple, il fallait composer par la pensée une série de loci ou de lieux, en attachant à chaque partie de chaque bâtiment un peu de ce qu'on voulait se rappeler. On devait associer les idées abstraites à des images, qu'on plaçait ensuite mentalement à des endroits précis, dans des lieux précis, qui pouvaient être imaginaires. On n'avait qu'à parcourir toutes les pièces du bâtiment réel ou imaginaire lorsqu'on voulait former un souvenir 
complet ${ }^{43}$. Flaubert donne une version amusante de cette méthode dans Bouvard et Pécuchet.

Ils prirent comme base mnémotechnique leur propre maison, leur domicile, attachant à chacune de ses parties un fait distinct, et la cour, le jardin, les environs, tout le pays, n'avaient plus d'autre sens que de faciliter la mémoire. Les bornages dans la campagne limitaient certaines époques, les pommiers étaient des arbres généalogiques, les buissons des batailles, le monde devenait symbole. Ils cherchaient, sur les murs, des quantités de choses absentes, finissaient par les voir, mais ne savaient plus les dates qu'elles représentaient ${ }^{44}$.

Bouvard et Pécuchet ont perdu de vue la chronologie globale de ce qu'ils essaient de mémoriser, ce qui fait qu'ils n'arrivent pas à en reconstituer l'essence. Avec le bâton de Belleau, ils n'auraient pas fait fausse route.

Un autre exemple de ce procédé mnémonique se trouve dans L'Étranger d'Albert Camus, où Meursault arpente sa cellule et essaie de faire une énumération complète de ce qui se trouvait dans sa chambre. Il s'agit de composer des souvenirs détaillés qui lui permettront de supporter son manque de liberté 45 .

Cette méthode mnémotechnique, correctement utilisée, repose sur le concept de la fragmentation temporaire: on divise son savoir afin de mieux le restituer ensuite. C'est une idée qui est importante dans La Bergerie, avec ses morceaux de prose et de vers, et les indications fréquentes que les morceaux ne sont pas complets. Dans La Bergerie de 1572, il s'agit souvent de fragments déjà parus ailleurs - avec seule l'idée d'une journée comme cadre. Le narrateur de La Bergerie de 1565, ayant commencé à décrire le bâton, observe: «Le tige de ce baston se met en quatre pieces qui servent de quatre fleutes à neuf trous fort belles \& bien compassees» (p. 111, l. 10-12). On pense dans ce passage rythmique à la baguette d'un chef d'orchestre. Un ensemble est divisé en morceaux, fragmenté, d'où une prolifération textuelle évoquée en partie à travers les chiffres. Il faut se rappeler aussi que la musique est, selon une tradition fort ancienne, dotée du pouvoir de guérir, et cela sera un thème capital

43 Pour ce système chez Quintilien, voir Frances A. Yates, The Art of Memory, p. 3.

44 Édition d'Éd. Maynial, Classiques Garnier (Paris: Garnier, 1965), p. 149-150.

45 Nous avons utilisé l'édition de Ray Davison, Routledge Foreign Literature Classics (Londres et New York: Routledge, 2007; première édition Routledge 1988), II. 2, p. 123. Meursault observe: «au bout de quelques semaines, je pouvais passer des heures, rien qu'à dénombrer ce qui se trouvait dans ma chambre. Ainsi, plus je réfléchissais et plus de choses méconnues et oubliées je sortais de ma mémoire.» 
dans «Les Amours de David et de Bersabee». Quatre jeunes bergers se servent de leurs flûtes pour chanter des «baisers»; ensuite ils donnent au narrateur «le double de leurs baisers» (p. 111, l. 17-18). L'idée d'un exemplaire écrit original qui est à la base de la représentation appuie l'exactitude de ce que le narrateur est censé recopier. Le papier conserve la parole et la musique ${ }^{46}$. Il produit à l'aide du bâton une impression de permanence qui réconforte particulièrement dans une époque tourmentée.

À tout prendre, lorsque Belleau décrit les châteaux à Joinville et le passé de quelques-uns des individus qui y vivent, il recrée non seulement les endroits qu'il connaît bien, fréquentés par les Guises, mais de véritables lieux de mémoire ${ }^{47}$. Il nous fait penser de surcroît aux guides topographiques et historiques de l'époque, qui tracent le développement des rapports spatiaux entre les lieux et les individus ${ }^{48}$. Ce sont ces rapports que le bâton est censé mesurer. Lorsque tout est soigneusement calibré l'homme peut se sentir protégé et guéri.

\subsection{Une colonne triomphale}

Vers le début de La Bergerie de 1565, le bâton a une espèce d'équivalent de grande dimension, sous forme d'une colonne, qui montre que Belleau s'intéresse dans son texte non seulement aux mots qu'on se remémore, mais aussi à la mémoire eidétique, que la mémoire verbale et linéaire arrive souvent à déclencher. Il imagine qu'une colonne est construite pour le duc de Guise. L'évocation de cette colonne est moins réaliste que celle du tombeau de Claude de Lorraine, qui existait vraiment (II, p. 24-25); sur elle sont suspendues des armes, dont des casques symbolisant des atrocités récentes et appelant à la vengeance, et des lieux de défaites dont l'abondance est désignée par l'anaphore «Cent $\&$ cent

46 Sur le processus d'échange de l'écriture et de la parole, de la lecture et de l'écriture, voir Nathalie Dauvois, Mnémosyne, p. 90.

47 Pour ce terme, voir Pierre Nora, éd., Les Lieux de mémoire, Bibliothèque illustrée des histoires, 7 vols (Paris: Gallimard, 1984-1992). Voir aussi François Hartog, «Mémoire, histoire et lieux de mémoire», Memoria e memorie, Convegno internazionale di studi, Roma, 18-19 maggio 1995, Accademia Nazionale dei Lincei, éd. Lina Bolzoni, Vittorio Erlindo, Marcello Morelli, Centro Internazionale di Cultura «Giovanni Pico della Mirandola», Studi Pichiani, 6 ([Florence:] Olschki, 1998), pp. 87-106.

48 Voir Elisabeth Hodges, «Representing Place in Corrozet's Antiquitez de Paris», French Studies, 62 (2008), 135-149. Voir aussi Chantal Liaroutzos, Le Pays et la mémoire: Pratiques et représentations de l'espace français dans les œuvres de Gilles Corrozet et Charles Estienne (Paris: Champion, 1998), et Cynthia Skenazi, Le Poète architecte en France: constructions d'un imaginaire monarchique (Paris: Champion, 2003). 
[...] Cent \& cent» (p. 31-32, v. 146-166) 49 . À chaque objet attaché à la colonne sont associés des souvenirs de la vaillance du prince. Un énorme monument se construit sous l'œil du lecteur, à partir d'une énumération qui oppose les vertus du duc d'une part et les effets de la vieillesse et de la mort d'autre part:

Afin que d'age, en age, on remarque la gloire,

La bonté, la vertu, l’honneur, \& la victoire

De ce grand Chevalier, qui surmonta l'effort

Des armes, du tombeau, des ans, \& de la mort. (p. 32, v. 167-170)50

L'hypotypose consacrée à la colonne montre comment la mémoire verbale et linéaire peut faire appel à ou être complétée par la mémoire visuelle. Grâce aux mots qui prolifèrent, mais grâce aussi aux images qu'ils créent, la mémoire du duc triomphera du temps. Le poème se termine pourtant sur le mot «mort», qui est précisément ce que le tombeau paraît vaincre. Tout en affirmant la survie du duc, le mot nous rappelle la nature transitoire de la vie ainsi que la fragilité de la mémoire humaine et de la gloire. Les vertus apotropaïques de la colonne sont grandes, mais sont limitées par la brièveté même de la vie humaine.

\subsection{Un bâton thérapeutique}

Avant tout, pourtant, Belleau met en valeur le pouvoir thérapeutique de l'écriture. Un long passage dans la description du bâton ingénieux, passage trop long pour être cité ici en entier, décrit la poignée de cet instrument:

L'entour de cette poignée est tracé de sept lignes \& sept espaces desquelles y en a six de mesme longueur, la septiesme est plus longuette que les autres $\&$ c'est celle qui monstre $\&$ marque les heures devant midy en descendant, $\&$ celles qui suyvent apres en montant. Les douze zignes [sic] du Zodiaque sont compris dedans les six espaces, six en montant jusques au solstice d'Esté, \& six en devalant. Ces six lignes sont tirees égalles en longueur $\&$ paralelles, mi-parties d'une ligne plus courte, puis entre ces divisions, qui

49 Nathalie Dauvois parle chez Ronsard de «cette parole réitérée par l'anaphore, conjuguée au présent du toujours, [...] consacrée à la réitération perpétuelle» (Mnémosyne, p. 67).

$\mathbf{5 0} \mathrm{Cf}$. le vers 5 du sonnet ronsardien qui commence «Afin qu'à tout jamais de siecle en siecle vive» (Sonnets pour Helene, II. 2): «Afin que d'âge en âge à noz neveux arrive[...]». 
sont douze, y a encores deux petites lignes $\&$ trois espaces, qui ne sont que marques ou points, lesquelles contiennent entre-elles l'espace de cinq jours, lesquels multipliez six fois, font trente jours ou trente degrez, que tient chacune espace ou signe du Zodiaque, lesquels mis ensemble, font le cours Solaire, ou un an entier. II y a d'autres lignes tortues qui tournent obliquement, marquees $\&$ tirees sur celles qui tombent à plom, par elles se cognoist la hauteur $>,<$ du Soleil chaque heure, chaque jour, $\&$ chaque signe, selon le cours d'iceluy. (II, p. 109, l. 6 - p.110, l. 24)

Avec des précisions mathématiques éblouissantes, voire amusantes, le passage suggère comment le bâton donne le moyen de se repérer dans le temps, de suivre l'écoulement des heures, des mois, de l'année, grâce à des marques et des lignes tracées sur la surface de l'outil. Un autre passage tout aussi impressionnant montrera combien l'instrument est utile lorsqu'il s'agit de mesurer l'espace (p. 118, l. 1 - p. 119, l. 17). Le bâton permet à celui qui s'en sert de se sentir à l'aise parce que cet individu sait exactement où et à quel moment de l'année il se trouve. Comme de nos jours on met la date sur ses photographies afin de mieux s'y retrouver dans les souvenirs qu'elles rappellent, le bâton facilite le classement des images retenues par la mémoire et contribue ainsi au bien-être et aux facultés cognitives de son possesseur. Le texte de Belleau reconstitue l'instrument et ses fonctions et invite le lecteur à prendre racine, à se mettre à l'aise, à profiter d'un instant de calme représenté par le château et ses environs qui ont été si soigneusement décrits dans le reste du texte ${ }^{51}$. Dans une époque troublée ces conseils transmis par la plume du poète sont particulièrement bénéfiques ${ }^{52}$.

La Bergerie est un texte qui examine entre autres la gloire et la mémoire. Le passage consacré au bâton, ainsi que celui qui dépeint la colonne, montrent à quel point l'identité de chacun est liée à ses souvenirs d'un endroit particulier, tel que Joinville. Malgré les doutes exprimés de temps à autre dans le reste de $L a$ Bergerie sur la durabilité de la gloire, ils suggèrent que la trace de chaque homme survivra au moins aussi longtemps que les lieux qu'il a connus. Ils constituent même une thérapie: se souvenir est essentiel au bien-être, à l'équilibre, à la contemplation de l'avenir ${ }^{53}$.

51 Comparer la façon dont Du Bellay, dans Les Regrets, recherche le séjour. Voir Yvonne Bellenger, Du Bellay: Ses «Regrets» qu'il fit dans Rome (Paris: Nizet, 1975).

52 Voir Jean Braybrook, «Space and Time in Remy Belleau's Bergerie», Bibliothèque d'humanisme et Renaissance, 57 (1995), 369-380.

53 Pour la façon dont la mémoire prépare l'avenir, voir Jean-Yves et Marc Tadié, Le Sens de la mémoire (Paris: Gallimard, 1999), p. 11. 


\subsection{Des remèdes de papier}

La Bergerie offre à divers personnages des textes - à première vue disparates, souvent incomplets, et censés être écrits parfois sur des bouts de papier - qui les encourageront et les aideront à retrouver le calme nécessaire à la méditation ${ }^{54}$. Belleau espère qu'ils auront les mêmes effets sur le lecteur. Bon nombre de ces textes, surtout en 1565, chantent la louange d'une grande famille, celle des Guises, qui a aidé Belleau à recouvrer la santé et à affirmer son identité. Il est vrai que les personnages de «La Seconde Journée» tirent souvent un poème de leur poche. La structure de l'ensemble de La Bergerie de 1572 est devenue plus décousue. Avec l'autodénigrement traditionnel dans les épîtres dédicatoires, mais en même temps avec un grain de vérité, Belleau explique à «Monseigneur Loys Monsieur de Lorraine» (dont l'identité n'est pas certaine) que

voulant recoudre ces inventions mal cousues, mal polies et mal agencées, sans l'esperer, je trouve un livre ramassé de pièces rapportées, chose véritablement que n'a membre, ny figure qui puisse former un corps entier et parfaict ${ }^{55}$. (IV, p. 147)

Cependant, sur la manière dont il convient de percevoir cette structure décousue, nous ne sommes pas tout à fait d'accord avec Frank Lestringant, puisque notre interprétation est moins pessimiste que la sienne. Ayant souligné la désinvolture et l'arbitraire avec lesquels de nouveaux poèmes sont intégrés à la trame générale de la deuxième édition de La Bergerie ${ }^{56}$, il conclut, «De la Bergerie de 1565 à celle de 1572, la dégradation de la situation politique en France, avec les seconde et troisième guerres de religion et la montée des périls qui aboutira bientôt à la Saint-Barthélemy, est sans doute pour quelque chose dans ce repli de l'imagination pratique sur un songe de papier» (p. 249).

Il est vrai que le bâton et la colonne sont de l'édition de 1565; mais au lieu de voir un repli et un goût de l'irréel dans celle de 1572, nous dirions au contraire que Belleau veut renforcer le message représenté par le bâton et offrir ouvertement des textes, ou des morceaux de texte, capables d'aider

54 Voir Daniel Ménager, La Renaissance et le détachement, Études et essais sur la Renaissance, 91 (Paris: Classiques Garnier, 2011).

55 On comparera Montaigne au début de son essai «De l'amitié»: «Que sont-ce icy aussi à la verité que crotesques et corps monstrueux, rappiecez de divers membres, sans certaine figure, n'ayants ordre, suite ny proportion que fortuite?» (Essais, éd. Jean Balsamo, Michel Magnien et Catherine Magnien-Simonin, Bibliothèque de la Pléiade (Paris: Gallimard, 2007), I. 27, p. 189).

56 «Les Amours pastorales de Daphnis et Chloé», p. 248. 
le lecteur à faire face à des situations diverses et souvent douloureuses ${ }^{57}$. Son but est très pratique. Certes, le fait que ces textes aient survécu semble fortuit et la mémoire humaine est présentée comme fragile. Belleau met pourtant l'accent sur l'accessibilité de ces textes, qu'on peut porter sur soi, qu'on n'est pas obligé d'aller consulter dans une bibliothèque. On choisit des fragments à son goût, capables de provoquer certaines émotions; on compose avec ces morceaux son vade-mecum personnel. On les consulte à n'importe quel moment de la journée. Le fait que les personnages de Belleau aient des poèmes cachés dans leurs vêtements souligne par conséquent qu'ils ont des ressources littéraires à portée de main, prêtes à être utilisées même ou surtout lorsque les circonstances ne facilitent pas une lecture ininterrompue. Ce sont les hypomnemata dont parle Daniel Ménager dans un article intitulé «L'Autoportrait», écrit pour Le Magazine littéraire ${ }^{58}$. De façon similaire Montaigne offre en fait des bribes de texte, des citations, à ses lecteurs. Qui plus est, dans le chapitre «De trois commerces» (III. 3) il explique comment il voyage toujours, même ou surtout pendant les guerres civiles, avec des livres qu'il feuillette «à pieces descousues»: «ils sont à mon costé pour me donner du plaisir à mon heure» ${ }^{59}$.

Belleau suggère de même que des textes distribués en apparence au hasard et portant sur des sujets divers aideront à rendre la vie plus supportable et que la mémoire s'appuiera en partie sur ces textes, en partie sur l'ordre et la mesure représentés par le bâton, pour créer des instants de bonheur et pour guérir l'esprit et l'âme, ce qui est crucial pendant la guerre. Cultiver l'ordre en soi-même ou dans une petite communauté est un moyen de promouvoir la paix et de remédier aux maux que le début du texte évoque avec insistance. L'idée d'un objet conjuratoire, prophylactique n'est certes pas neuve; mais la manière dont Belleau la rend concrète avec sa description minutieuse du bâton et de la colonne est saisissante ${ }^{60}$. De façon similaire, le recueil des Amours et nouveaux eschanges des pierres precieuses présentera des gemmes qui sont des lieux de mémoire mais qui peuvent aider aussi à oublier la haine et le désir de se venger (VI, p. 56, v. 73-76) ${ }^{61}$.

$57 \mathrm{Cf}$. le recueil de lieux communs qu'un écolier du XVIe siècle composait en lisant; voir la note 2 ci-dessus.

58464 (mai 2007), p. 38-40. Il s'agit d'un dossier sur Montaigne. Nous remercions Agnès Calatayud pour cette référence.

59 Édition de Jean Balsamo, Michel Magnien et Catherine Magnien-Simonin, p. 869.

60 On comparera, sur la fonction conjuratoire du barde, Robert Graves, The Crowning Privilege: The Clark Lectures, $1954-1955$ (Londres: Cassell, 1955; New York: Doubleday, 1956). C'est Susan Reynolds qui a attiré notre attention sur ce livre.

61 Voir Evelien Chayes, L'Éloquence des pierres précieuses, p. 135. 
Il y a cependant un domaine dans lequel le bâton ne serait peut-être pas toujours efficace: celui de l'amour. Les deux prochains chapitres concerneront ainsi le sujet incontournable qu'est la passion amoureuse, capable de perturber complètement ceux qui en font l'expérience (ainsi que le montre par exemple Ronsard, en particulier dans ses Sonnets pour Helene). Même les Grands sont touchés: Belleau décrira un roi biblique éperdument amoureux et oublieux de tous ses principes. Quels remèdes Belleau propose-t-il donc à la souffrance causée par l'amour? 